\title{
A fracture parameter for welded structures with residual stresses
}

\author{
Y.-C. Hou, J. Pan
}

\begin{abstract}
In this paper, a finite element procedure to determine a fracture parameter $J_{\mathrm{ld}}$ is presented for welded structures with consideration of residual stresses. The method is based on the energy difference of two cracked solids with slightly different crack sizes. Our computational results show that $J_{\text {ld }}$ and the $J$ integral agree well for a cracked plate without consideration of residual stresses. When the residual stresses are considered, the values of $J_{\text {ld }}$ for different contours close to the crack tip in the cracked plate subject to remote tensile stresses are in good agreement. The computational results also indicate that for the given residual stress distribution, the values of $J_{\text {ld }}$ with consideration of residual stresses are lower than those without consideration of residual stresses for the cracked plate subject to large remote tensile stresses.
\end{abstract}

\section{1}

\section{Introduction}

The path independent $J$ integral proposed by Rice (1968a, b) for deformation plasticity (nonlinear elastic) materials has been used widely used to characterize the crack-tip stress and strain fields. The $j$ integral represents the energy release rate for deformation plasticity (nonlinear elastic) cracked solids. The energy release rate is defined as the decrease of the potential energy per unit area of crack advance. Begley and Landes (1972a, b) obtained the values of $J$ from the load-displacement curves of multiple cracked specimens based on the concept of the energy release rate. To compute the values of $J$ by finite element methods, Parks (1974) and Hellen (1975) proposed the virtual crack extension method based on the change of the finite element stiffness matrix with respect to the crack length for linear elastic materials.

Parks (1977) generalized the stiffness derivative finite element technique to determine the values of $J$ for nonlinear elastic materials. The generalized method is based on the energy difference of two cracked bodies with slightly different crack sizes. It should be noted that this

Communicated by S. N. Atluri, 28 April 1998

Y.-C. Hou, J. Pan

Department of Mechanical Engineering and Applied Mechanics, The University of Michigan, Ann Arbor, MI 48109, USA.

E-mail: jwo@engin.umich.edu.

Ph: (734) 764-9404; Fax: (734) 647-3170

Correspondence to: J. Pan technique requires only one nonlinear finite element solution. deLorenzi (1982) derived an analytical expression for the energy release rate by using the virtual crack extension method from a continuum mechanics viewpoint. Shih et al. (1986), Moran and Shih (1987), and Nikishkov and Atluri (1987a, b) developed the domain integral method to evaluate the $J$ integral. A more detailed discussion of various methods can be found in Atluri (1997). The expression for the domain integral method is consistent with that obtained by deLorenzi (1982) when the smooth function $q$ used in the domain integral method is interpreted as the normalized virtual displacement. Atluri et al. (1984) presented incremental path-independent integrals for elastic-plastic materials. Both quasi-static and dynamic fracture conditions were considered in their study. Brust et al. (1986) examined a fracture parameter $T^{\star}$ after a cycle of loading and unloading to zero load followed by reloading. Wang et al. (1997) examined the $T^{\star}$ parameter in the context of stable crack growth in aircraft structures. The results of their combined numerical and experimental study showed that $T^{\star}$ accurately predicted the fracture behavior under unloading and reloading conditions. Nakagaki et al. (1989) conducted a combined experimental and analytical study of ductile crack growth in tungsten inert gas (TIG) weld stainless steel specimens. In their finite element analysis, they showed that $J$ is path dependent due to the stress and strain nonproportionality associated with the local unloading due to crack growth, whereas $\Delta T_{p}^{*}$ and $\hat{J}$ are path independent.

During the welding processes, weld metals and, possibly, adjacent base metals are heated above the melting temperature and then solidify. After the welding processes, large residual stresses may develop in the welds and the heat affected zones (HAZs) due to thermoelastic-plastic deformation. Under service loading conditions, cracks may be initiated and grown. When the plastic deformation becomes extensive near the crack tips due to large service loads, elastic-plastic fracture parameters are needed to characterize the fracture processes near the crack tips in these welded structures with residual stresses. Therefore, we proposed a finite element procedure to determine a potential fracture parameter $J_{\mathrm{ld}}$ for welded structures with residual stresses. The procedure is based on the concept of the energy difference under the load-displacement curves of the two cracked solids with slightly different crack sizes.

Specifically, we perform a thermoelastic-plastic finite element analysis of the multi-pass welding process of two stainless steel plates under plane strain conditions. After the residual stresses are determined, a crack is introduced 
in the welded plate along the symmetry plane. Then remote tension is applied at the far ends of the welded plate. Because of the existence of residual stresses, the $J$ integral for deformation plasticity (nonlinear elastic) materials may not be appropriate to characterize the crack-tip field.

However, fracture parameters are needed to characterize the fracture processes near the tip. A local fracture parameter such as the crack tip opening angle (CTOA), a computationally convenient parameter, may be used (for example, see Hou et al., 1996). Nevertheless, a fracture parameter based on conveniently measurable quantities such as the loads and displacements in experiments is more desirable. Therefore, we compute the values of $J_{\text {ld }}$ by the proposed finite element procedure for the cracked plate with consideration of residual stresses and explore the implications of $J_{\mathrm{ld}}$ for characterization of the fracture processes near the crack tip.

\section{2}

\section{Formulation}

The $J$ integral (Rice, 1968a) for nonlinear elastic materials can be expressed as

$J=\int_{\Gamma}\left(W n_{1}-t_{1} \frac{\partial u_{i}}{\partial x_{1}}\right) \mathrm{d} s$

where $\Gamma$ represents a counterclockwise contour around the crack tip from the lower crack face to the upper crack face, $W$ is the strain energy density function, $n_{1}$ is the $x_{1}$ component of the unit outward normal vector to contour $\Gamma, t_{i}$ are the components of the traction, $u_{i}$ are the components of the displacement vector, and $\mathrm{d} s$ is the differential arc length of $\Gamma$. Here the summation convention is adopted for repeated indices. It has also been shown by Rice (1968b) that the $J$ integral represents the energy release rate for deformation plasticity (nonlinear elastic) cracked solids. As shown in Fig. 1(a), we consider a cracked plate of unit thickness with the crack length $a$. In the figure, $P$ represents the load and $u$ represents the loadpoint displacement.

For nonlinear elastic materials, $J$ can be expressed as

$J=-\frac{\partial \Pi}{\partial a}$

Where $\Pi$ represents the potential energy expressed as

$\Pi=U-P u$.

Here $U$ is the strain energy stored in the body and is the area under the load-displacement curve. For nonlinear elastic materials,

$\Pi=U-P u=-U^{*}$

where $U^{*}$ represents the complementary strain energy as

$U^{*}=\int_{0}^{P} u \mathrm{~d} P$.

Thus, if the cracked plate is under load-controlled conditions, $J$ is given by

$J=\left(\frac{\partial U^{*}}{\partial a}\right)_{P}=\int_{0}^{P}\left(\frac{\partial u}{\partial a}\right)_{P} \mathrm{~d} P$.
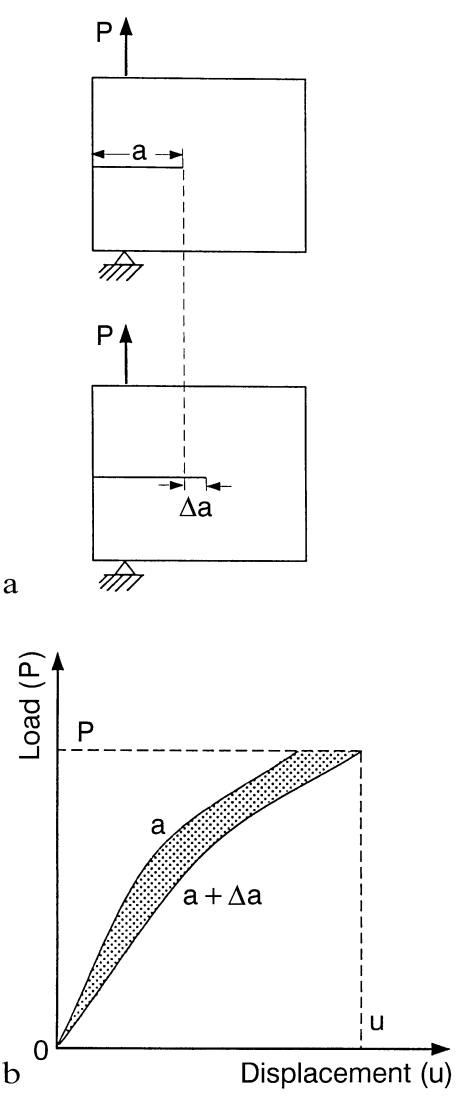

Fig. 1. a Two cracked bodies with different crack lengths by $\Delta a$. b Nonlinear load-displacement curves for the two cracked bodies

Now, as shown in Fig. 1(a), we consider two cracked bodies with the identical geometry except that the crack length of the second body is longer than that of the first one by $\Delta a$. We can schematically plot the load-displacement curves for the two cracked bodies in Fig. 1(b). The shaded area $\Delta A$ as shown in Fig. 1(b) is the complementary strain energy difference of the two cracked bodies.

Based on the concept discussed above, we consider a parameter $J_{\text {ld }}$ to represent the energy difference of two cracked bodies, normalized by a small difference of crack sizes, subject to the same loading history, even when the cracked bodies have significant amount of residual stresses. Therefore, $J_{\text {ld }}$ for elastic-plastic materials with residual stresses is defined as

$J_{\mathrm{ld}}=\lim _{\Delta a \rightarrow 0} \frac{\Delta A}{\Delta a}$.

Our finite element procedure to determine $J_{\text {ld }}$ is based on the principle of virtual work. In the absence of the body force, the principle of virtual work is written as

$\int_{V} \sigma_{i j} \delta \epsilon_{i j} \mathrm{~d} V=\int_{S} T_{i} \delta u_{i} \mathrm{~d} S$

where $\sigma_{i j}$ are the stresses, $\delta \epsilon_{i j}$ are the virtual strains, $T_{j}\left(=n_{i} \sigma_{i j}\right)$ are the components of the surface traction and $\delta u_{i}$ are the virtual displacements. Since we consider elastic-plastic materials, we compute the finite element displacement incrementally. The principle of virtual work for 
expansion from a known equilibrium state can be written as

$$
\int_{V}\left(\sigma_{i j}+\Delta \sigma_{i j}\right) \delta \epsilon_{i j} \mathrm{~d} V=\int_{S}\left(T_{i}+\Delta T_{i}\right) \delta u_{i} \mathrm{~d} S
$$

where $\Delta($ ) denotes the incremental quantity from the known state.

Then, for finite element applications, Eq. (9) can be rearranged in the matrix form as

$$
\begin{aligned}
& {[\boldsymbol{\delta} \mathbf{u}]^{\mathrm{T}}\left(\sum_{m=1}^{M_{c}} \int_{V^{(m)}}\left[\mathbf{B}^{(m)}\right]^{\mathrm{T}}\left[\mathbf{C}_{\mathrm{ep}}^{(m)}\right]\left[\mathbf{B}^{(m)}\right] \mathrm{d} V^{(m)}[\Delta \mathbf{u}]\right.} \\
& \quad-\sum_{e=1}^{E_{c}} \int_{S_{(e)}}\left[\mathbf{N}^{(e)}\right]^{\mathrm{T}}\left[\Delta \mathbf{T}^{(e)}\right] \mathrm{d} S^{(e)} \\
& \quad-\sum_{e=1}^{E_{c}} \int_{S_{(e)}}\left[\mathbf{N}^{(e)}\right]^{\mathrm{T}}\left[\mathbf{T}^{(e)}\right] \mathrm{d} S^{(e)} \\
& \left.+\sum_{m=1}^{M_{c}} \int_{V_{(m)}}\left[\mathbf{B}^{(m)}\right]^{\mathrm{T}}\left[\boldsymbol{\sigma}^{(m)}\right] \mathrm{d} V^{(m)}\right)
\end{aligned}
$$

where a superscript $\mathrm{T}$ denotes the transpose, $M_{c}$ is the number of elements for the entire body, and $E_{c}$ is the number of elements on the traction-prescribed surface. Here, [B] is the incremental strain-displacement matrix, $\left[\mathrm{C}_{\mathrm{ep}}\right]$ is the elastic-plastic constitutive matrix, $[\Delta \mathbf{u}]$ is the incremental nodal displacement vector, $[\mathrm{N}]$ is the interpolation function matrix, $[\Delta \mathrm{T}]$ is the incremental traction vector, $[\mathrm{T}]$ is the traction vector, and $[\boldsymbol{\sigma}]$ is the stress matrix. Since $[\boldsymbol{\delta} \mathbf{u}]^{\mathrm{T}}$ is an arbitrary vector, the term enclosed in the large parentheses in Eq. (10) must be zero. This leads to

$$
[\mathbf{K}][\Delta \mathbf{u}]=[\Delta \mathbf{F}]+[\mathbf{F}]_{\text {res }}
$$

where

$$
\begin{aligned}
{[\mathbf{K}]=} & \sum_{m=1}^{M_{c}} \int_{V^{(m)}}\left[\mathbf{B}^{(m)}\right]^{\mathrm{T}}\left[\mathbf{C}_{\mathrm{ep}}^{(m)}\right]\left[\mathbf{B}^{(m)}\right] \mathrm{d} V^{(m)} \\
{[\mathbf{F}]_{\mathrm{res}}=} & \sum_{e=1}^{E c} \int_{S^{(e)}}\left[\mathbf{N}^{(e)}\right]^{\mathrm{T}}\left[\mathbf{T}^{(e)}\right] \mathrm{d} S^{(e)} \\
& -\sum_{m=1}^{M c} \int_{V^{(m)}}\left[\mathbf{B}^{(m)}\right]^{\mathrm{T}}\left[\boldsymbol{\sigma}^{(m)}\right] \mathrm{d} V^{(m)} .
\end{aligned}
$$

Here, $[\mathbf{K}]$ is the elastic-plastic stiffness matrix, $[\Delta \mathbf{F}]$ is the vector of the prescribed incremental nodal forces, and $[\mathbf{F}]_{\text {res }}$ represents the residual or the out of balance nodal forces within the body at the end of each increment.

Suppose that an elastic-plastic finite element analysis has been performed on a given body of unit thickness with the crack length $a$. We have obtained the vector of incremental nodal displacement $[\Delta \mathbf{u}]$ at each load increment. As shown in Fig. 2, we select a ring of finite elements extending from the lower crack face to the upper crack face. The ring of elements is bounded by the inner contour $\Gamma_{0}$ and the outer contour $\Gamma_{1}$. Then at the end of each load increment, we perturb the crack length by increasing the $x_{1}$ coordinates of the nodal points within and along the

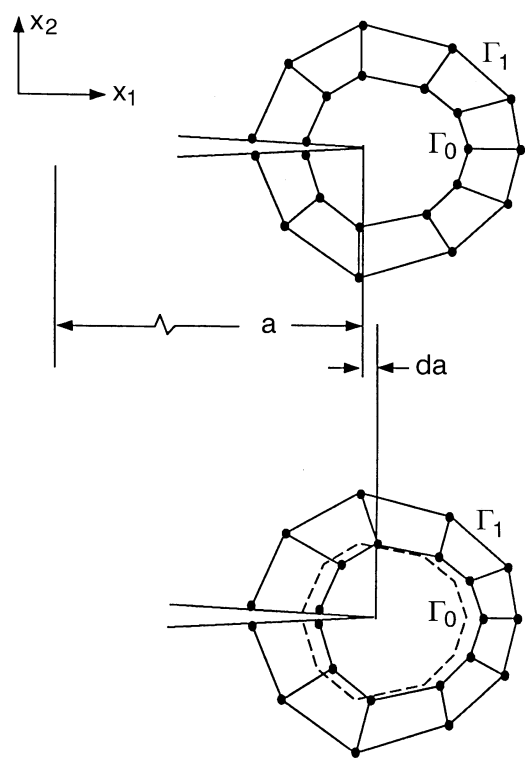

Fig. 2. Perturbation of the crack length by translating $d a$ for the nodal points within and along the inner contour $\Gamma_{0}$

inner contour $\Gamma_{0}$ by $d a$ under the same loading condition. When we perturb the crack length $a$, Eq. (11) becomes

$\frac{\partial[\mathbf{K}]}{\partial a}[\Delta \mathbf{u}]+[\mathbf{K}] \frac{\partial[\Delta \mathbf{u}]}{\partial a}=\frac{\partial[\Delta \mathbf{F}]}{\partial a}+\frac{\partial[\mathbf{F}]_{\text {res }}}{\partial a}$

Along the traction boundary, the applied traction is given and the traction is zero on the cracked faces. The first term on the right hand side of Eq. (12) is zero. To obtain the variation of the incremental nodal displacement vector with respect to the crack length $a, \partial[\Delta \mathbf{u}] / \partial a$, at each load increment, we assume that each material element have the same elastic-plastic matrix before and after the perturbation. Then, the variation in the load-point displacement is

$\left(\frac{\partial[\mathbf{u}]}{\partial a}\right)_{i}=\left(\frac{\partial[\mathbf{u}]}{\partial a}\right)_{i-1}+\frac{\partial[\Delta \mathbf{u}]}{\partial a}$

where the index $i$ represents the $i$ th increment. From Eq. (12),

$\frac{\partial[\Delta \mathbf{u}]}{\partial a}=-[\mathbf{K}]^{-1}\left(\frac{\partial[\mathbf{K}]}{\partial a}[\Delta \mathbf{u}]-\frac{\partial[\mathbf{F}]_{\text {res }}}{\partial a}\right)$.

In Eq. (14), $\partial[\mathbf{K}] / \partial a$ can be evaluated directly by differentiation of the elastic-plastic stiffness matrix $[\mathbf{K}]$ for the elements in the ring as shown in Fig. 2, see also Atluri (1997).

Alternatively, from a finite difference viewpoint, $\partial[\Delta \mathbf{u}] / \partial a$ in Eq. (14) can be calculated from $\partial[\boldsymbol{K}] / \partial \boldsymbol{a}$ and $\partial[\mathbf{F}]_{\text {res }} / \partial a$ by the forward finite difference method.

For example,

$\frac{\partial[\mathbf{K}]}{\partial a} \cong \frac{\Delta[\mathbf{K}]}{\Delta a}-\frac{1}{\Delta a}\left([\mathbf{K}]_{a+\Delta a}-[\mathbf{K}]_{a}\right)$.

When we take the finite difference method to compute $\partial[\mathbf{K}] / \partial a$, we need to perform two elastic-plastic computations for the cracked solid with two slightly different cracked sizes. 


\section{3}

\section{Numerical results}

To demonstrate the finite element procedure, we consider a welded plate with a crack subject to remote loading under plane strain conditions. Due to symmetry, a finite element model of a half of the welded plate is shown in Fig. 3(a). The plate has a thickness of $8.64 \mathrm{~mm}$. The shaded elements shown in Fig. 3(b) represent the welds deposited from the four welding passes. The combination of four 3-node triangles proposed by Nagtegaal et al. (1974) is used in our finite element model to account for the effect of incompressible or nearly incompressible behavior encountered in the fully plastic range. Note that the triangular elements are combined to form compatible quadrilateral elements. In our finite element analysis, the residual stresses are computed due to the four welding passes. A crack is then placed by releasing coupled nodal points sequentially from the bottom of the welded plate along the symmetry plane. The final crack length is $2.16 \mathrm{~mm}$ which is one quarter of the plate thickness. The numerical procedure to obtain the distribution of the residual stresses after the welding process and the introduction of the crack can be found in Hou et al. (1996). Figure 4 shows that the residual stress distribution in the $R$ (thickness) direction near the symmetry line $(Z=0)$ before the introduction of the crack. In the figure, $\sigma_{e}\left(=\left(3 \sigma_{i j}^{\prime} \sigma_{i j}^{\prime} / 2\right)^{1 / 2}\right.$ where $\sigma_{i j}^{\prime}$ are the deviatoric stresses $)$ represents the equivalent tensile stress, $\sigma_{R}$ and $\sigma_{z}$ are the in-plane normal stresses in the $R$ and $Z$ directions, respectively, $\sigma_{R Z}$ is the in-plane shear stress, and $\sigma_{T}$ represents the out-of-plane normal stress. These stresses are obtained at the integration points of the element closest to the symmetry line. In Fig. 4, we notice that the stress levels of the three in-plane stresses are significantly lower than that of the out-of-plane normal stress $\sigma_{T}$ which is the highest and has a magnitude from $340 \mathrm{MP}$ a to $740 \mathrm{MPa}$ through the thickness.

Three paths are selected for the computations of the $J$ integral for the crack as shown in Fig. 5(a). Note that the scale in the $R$ direction is magnified in Fig. 5 for the convenience of presentation. Also three different interior contours $\Gamma_{0}$ surrounding the crack tip are selected for determination of $J_{\mathrm{ld}}$ as shown in Fig. 5(b). The procedure outlined in the previous section is applied at each load

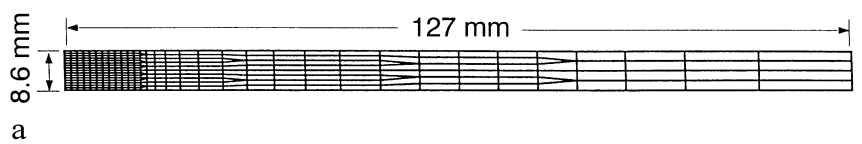

a

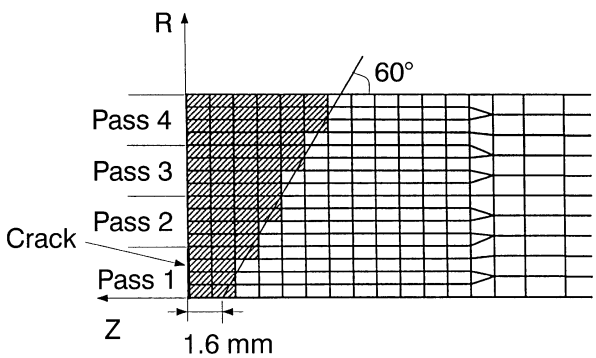

b

Fig. 3. a Finite element mesh for an $8.64 \mathrm{~mm}$ thick plate, b The four welding passes and the V-groove weld geometry

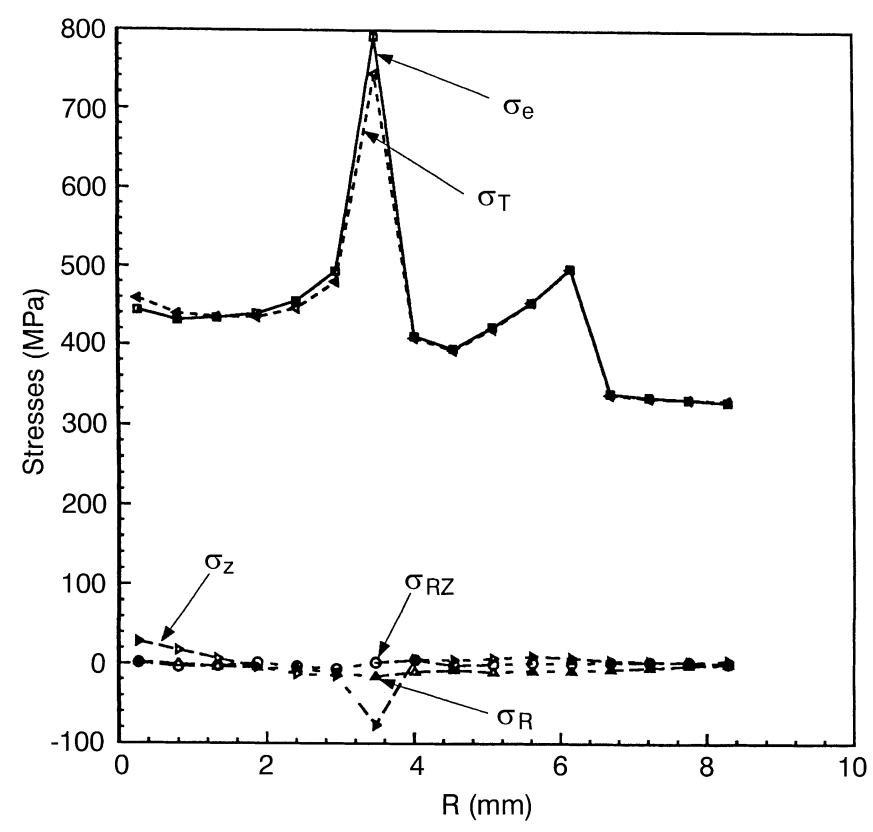

Fig. 4. Residual stress distribution in the $R$ direction near the symmetry plane

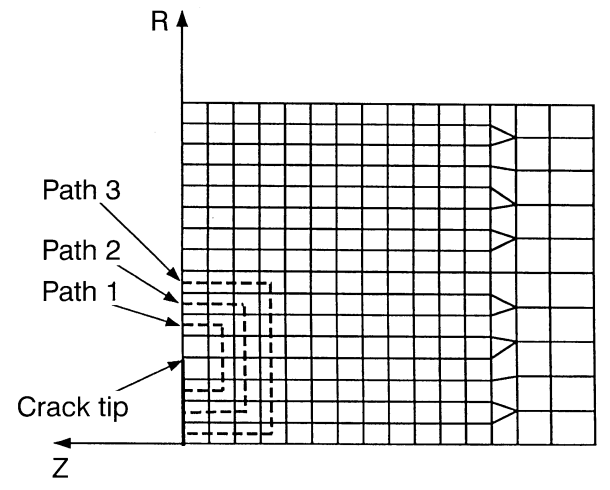

a

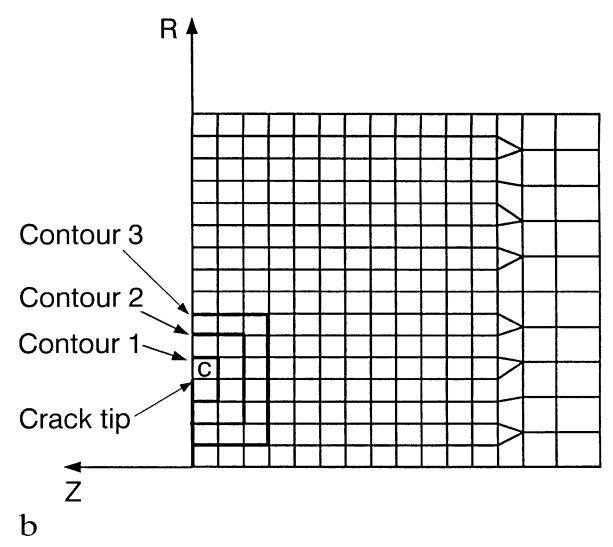

Fig. 5. a The $J$ integral paths, b The $J_{\text {ld }}$ contours

increment by advancing nodal points within and along each contour.

The material properties of the base metal and the weld metal follow those of Type 304 stainless steel in our finite element modeling (Hou et al., 1996). Our thermo-plastic behavior is based in an isotropic hardening model. The 
hardening model may have significant effects on prediction of residual stresses due to the welding processes. The strain hardening is assumed to be linear. The yield stress of this material at room temperature is $240 \mathrm{MPa}$.

If the residual stresses are not considered, the values of $J$ integral, $J^{1}, J^{2}$, and $J^{3}$ for different paths 1,2 , and 3 agree well at a given applied stress from our computations. In Fig. 6, the value of $J^{3}$ is plotted as a function of the applied tensile stress. Based on our finite element procedure, $J_{\mathrm{ld}}^{1}, J_{\mathrm{ld}}^{2}$ and $J_{\mathrm{ld}}^{3}$ for different contours 1,2 , and 3 are also computed and plotted in Fig. 6 . The values of $J_{\mathrm{ld}}^{1}, J_{\mathrm{ld}}^{2}$, and $J_{\mathrm{ld}}^{3}$ agree with each other within $3 \%$ as the applied stress increases to $370 \mathrm{MPa}$. Thus, $J_{\mathrm{ld}}$ shows path independence at the given applied stress when the residual stresses are not considered. It should be noted that $J_{\mathrm{ld}}$ represents the energy release rate for deformation plasticity (nonlinear elastic) materials. Thus, the values of $J_{\mathrm{ld}}$ should agree with the values of the $J$ integral throughout the loading history. Figure 6 shows a good agreement between the values of $J_{\mathrm{ld}}$ and the $J$ integral at the given applied tensile stress. The slight differences are possibly due to the different numerical methods to calculate the values of $J_{\mathrm{ld}}$ and $J$.

Figure 7 shows the values of $J_{1 d}^{1}, J_{1 d}^{2}$, and $J_{\text {ld }}^{3}$ for different contours 1,2, and 3 as functions of the applied tensile stress, when the residual stresses are considered. In the figure, the values of $J_{\text {ld }}^{2}$ show an excellent agreement with the values of $J_{1 \mathrm{~d}}^{3}$ throughout the loading history. However, the value of $J_{\text {ld }}^{1}$ is 15 percent below the value of $J_{\text {ld }}^{3}$ when the applied stress reaches $480 \mathrm{MPa}$. It should be noted that contour 1 is closer to the crack tip, the stress and strain values inferred from this contour may not be accurate.

Figure 8 shows the values of the $J$ integral for different paths and the value of $J_{\mathrm{ld}}^{3}$ as functions of the applied tensile stress, when the residual stresses are considered. When we account for the residual stresses induced by the welding

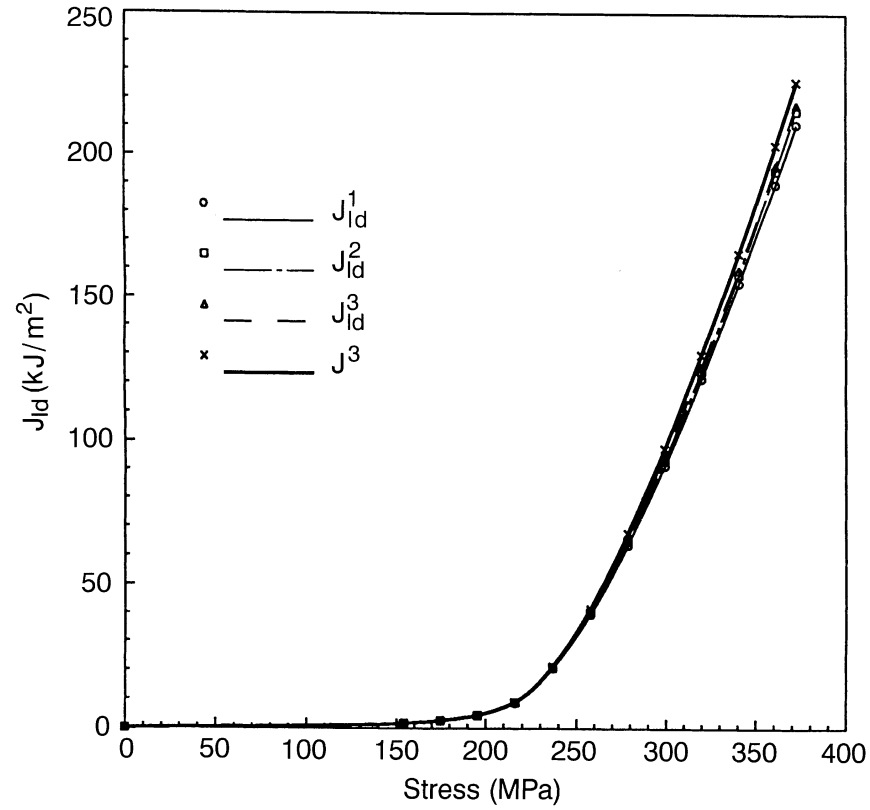

Fig. 6. The values of $J_{\mathrm{ld}}$ for different contours and the value of the $J$ integral for path $3, J^{3}$, as functions of the applied tensile stress without residual stresses

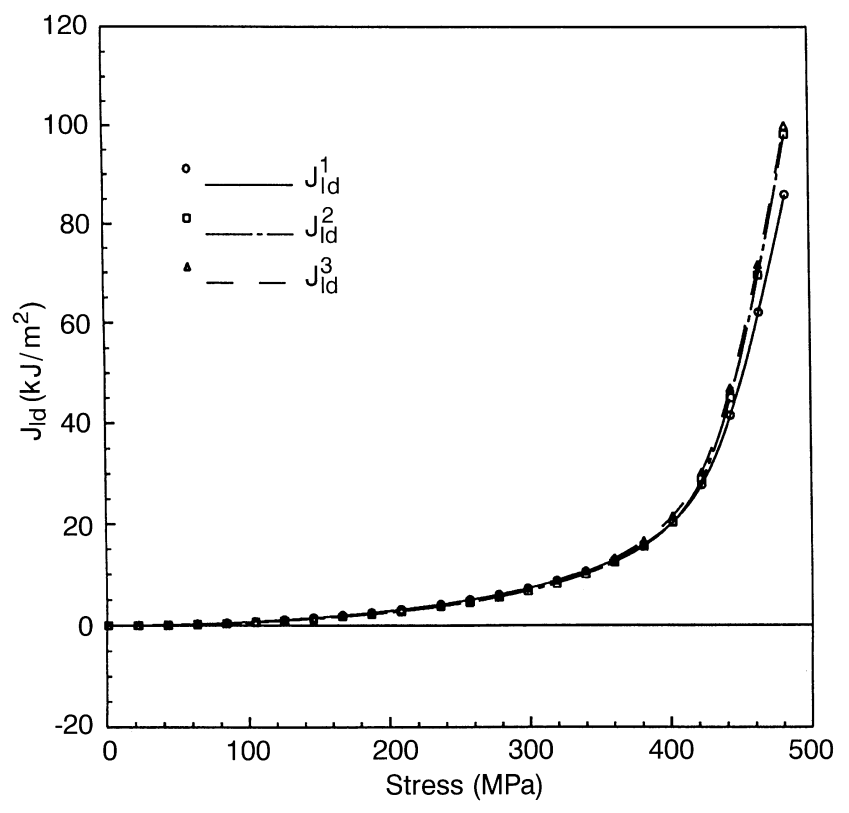

Fig. 7. The values of $J_{\text {ld }}$ for different contours as functions of the applied tensile stress with residual stresses

processes and the nonproportional loading due to the introduction of the crack, $W$ in Eq. (1) is taken as the stress work for calculation of the $J$ integral. In fact, the values of the $J$ integral shown in Fig. 8 represent the increase of the $J$ integral from the state where the applied tensile stress is zero. Due to the residual stresses, the path dependence of the $J$ integrals can be clearly seen in the figure. For comparison, $J_{\mathrm{ld}}^{3}$ is also shown in the figure. The link of $J_{\mathrm{ld}}$ with consideration of residual stresses to the crack-tip parameters such as the crack tip opening angle (CTOA) will be discussed later in the paper.

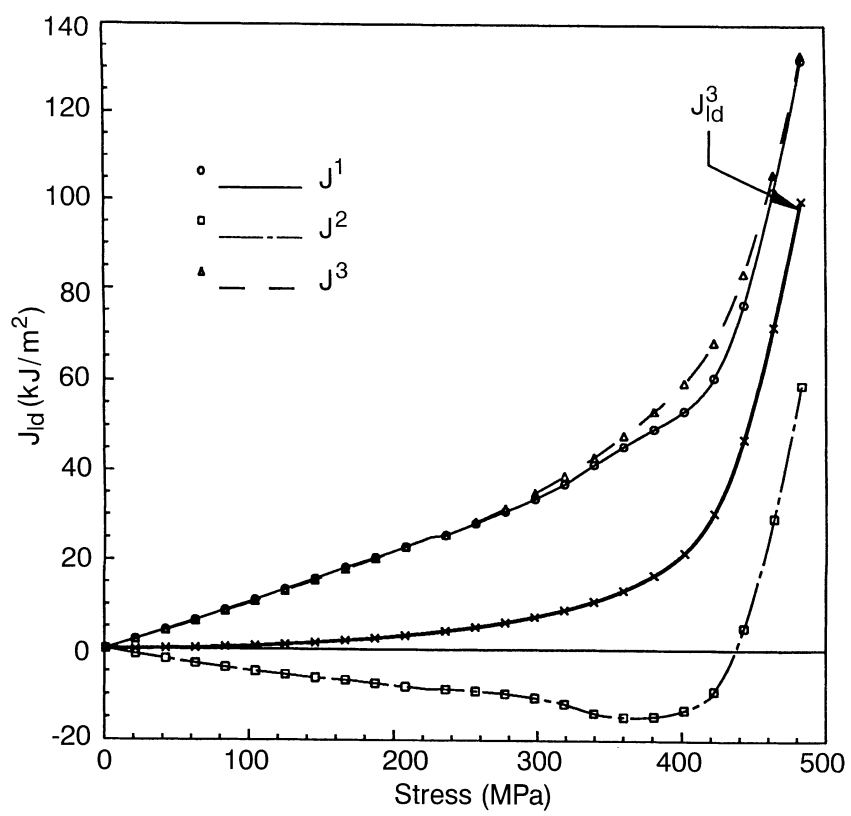

Fig. 8. The values of the $J$ integrals for different contours and the value of $J_{\text {ld }}^{3}$ for contour 3 as functions of the applied tensile stress with residual stresses 
Figure 9 shows the values of $J_{\text {ld }}^{3}$ obtained from different methods: the finite difference method and the elasticplastic stiffness differentiation method that we present here, when the residual stresses are considered. In the finite difference method, the size of $\Delta a$ used is about $10^{-4}$ of the size of the finite elements near the tip. The figure shows that the computed values of $J_{\text {ld }}^{3}$ obtained from the finite difference method and the elastic-plastic stiffness differentiation method are almost identical. Note that the values of $J_{\mathrm{ld}}^{3}$ are computed by perturbing contour 3 shown in Fig. 5(b).

Figure 10 shows the values of $J_{\mathrm{ld}}^{3}$, with and without consideration of residual stresses, as functions of the applied tensile stress. In general, we can see that the values of $J_{\text {ld }}^{3}$ with consideration of residual stresses are lower than those without consideration of residual stresses at large applied stresses. When the residual stresses are not considered, we see that the values of $J_{\text {ld }}^{3}$ increase substantially near $240 \mathrm{MPa}$ (the room temperature yield stress) due to large plastic deformation. However, we should keep in mind that the welding processes under plane strain conditions induce large residual stresses in the out-of-plane direction, as shown in Fig. 4. Then large elastic stress increments must be applied to the material elements to reach the yielding conditions following extremely nonproportional loading paths due to the applied remote in-plane tension.

We now examine the equivalent stress history of the element in the front of the crack tip, marked by $C$ in Fig. 5(b), to demonstrate the argument discussed above. Figure 11 shows that the current equivalent yield stress $\sigma_{y}$ and the equivalent stress $\sigma_{e}$ of the crack tip element as functions of the applied tensile stress. The figure shows that the current equivalent yield stress $\sigma_{y}$ is about $500 \mathrm{MPa}$ due to the welding processes before the tensile stress is

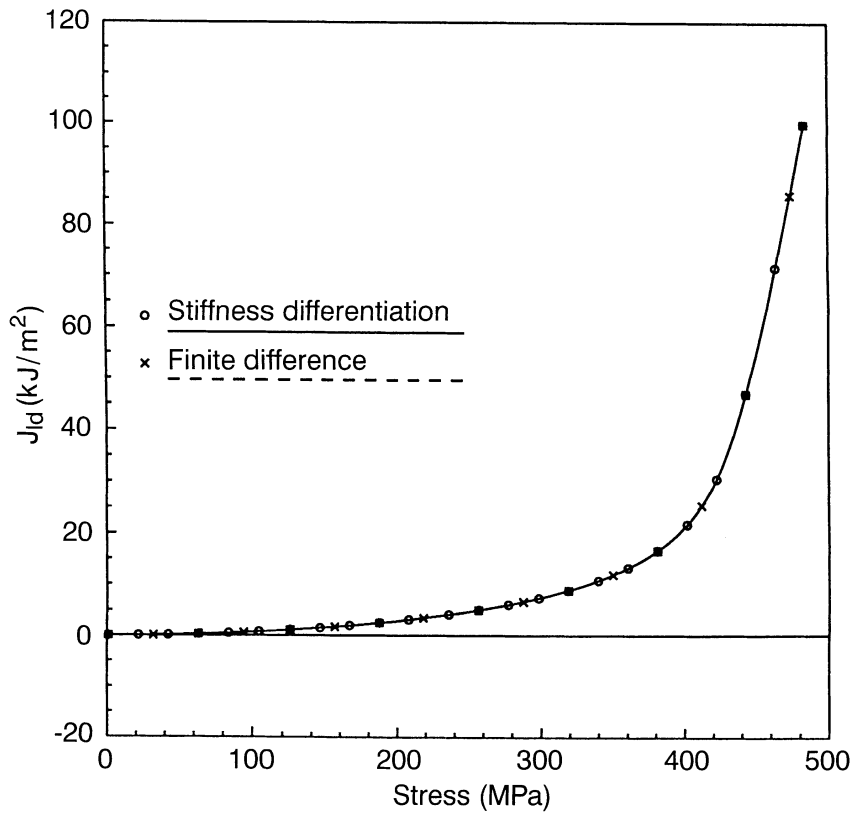

Fig. 9. The values of $J_{\mathrm{ld}}^{3}$, from the finite difference method and the stiffness differentiation method, as functions of the applied tensile stress with residual stresses

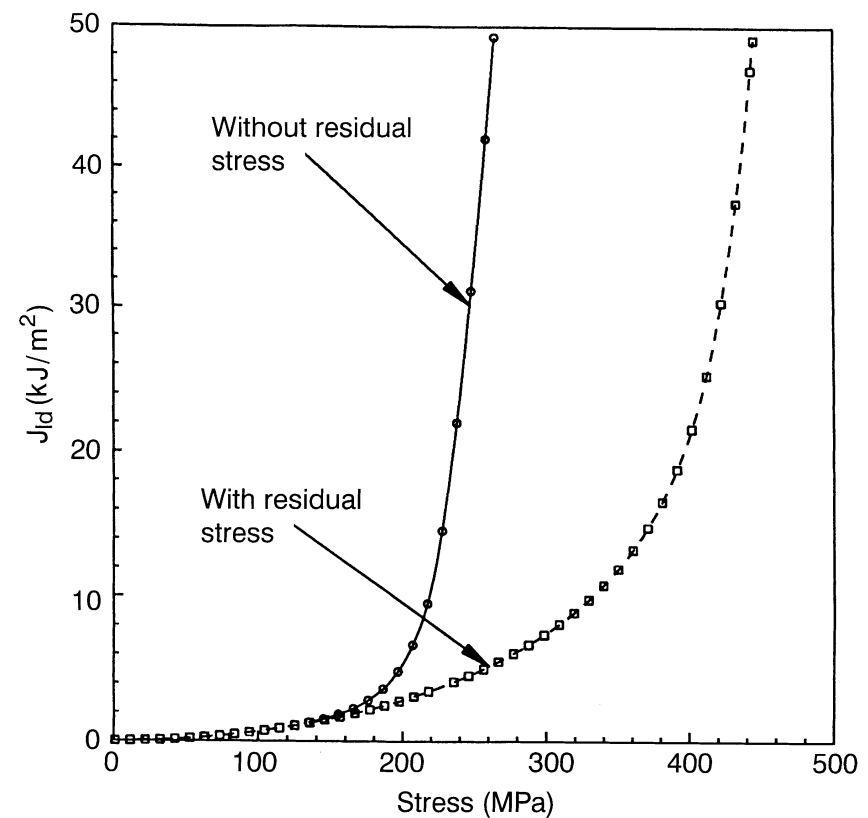

Fig. 10. The values of $J_{\text {ld }}^{3}$ as functions of the applied tensile stress with and without residual stresses

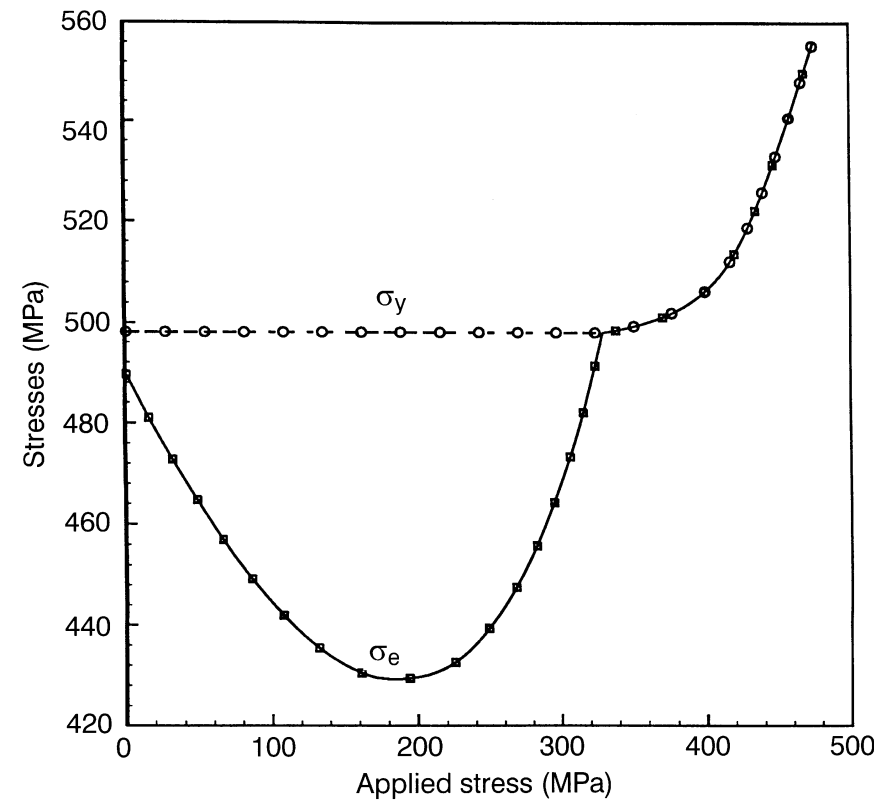

Fig. 11. The current equivalent yield stress $\sigma_{y}$ and the equivalent stress $\sigma_{e}$ of the crack tip element as functions of the applied stress

applied. The figure also shows that the applied stress initially causes further elastic unloading in the element. When the applied tensile stress is about $320 \mathrm{MPa}$, the equivalent stress of the element becomes equal to the current equivalent yield stress and the element becomes plastic again. When the applied tensile reaches $420 \mathrm{MPa}$, our computational results indicate that the displacement due to the applied stress becomes very large. Thus, the value of $J_{\text {ld }}$ with consideration of residual stress increases substantially near the applied stress of $420 \mathrm{MPa}$, as shown in Fig. 10. Therefore the values of $J_{\mathrm{ld}}$ with consideration of residual stresses are lower than those of $J_{\mathrm{ld}}$ without con- 
sideration of residual stresses when the applied tensile stresses are close to the room temperature yield stress $240 \mathrm{MPa}$. Figure 12 also shows the values of $J_{\mathrm{ld}}^{3}$, with and without consideration of residual stresses, as functions of the applied tensile stress up to $200 \mathrm{MPa}$. We can see that, when the applied stress is between 50 and $150 \mathrm{MPa}$, the values of $J_{1 \mathrm{~d}}^{3}$ with consideration of residual stresses are slightly larger than those without consideration of residual stresses.

Hou et al. (1996) used the crack tip opening angle (CTOA) as a computationally convenient parameter to characterize the deformation near the crack tip due to both the residual stress and the applied tensile stress. The crack tip opening angle (CTOA) is defined as

$\mathrm{CTOA}=2 \tan ^{-1}\left(\frac{\partial_{e}}{h_{e}}\right)$

where $\delta_{e}$ the displacement at the nodal point right behind the crack tip, and $h_{e}$ is the size of the element in the crackline direction right behind the crack tip.

Figure 13 shows the CTOAs, with and without consideration of residual stresses, as functions of the applied tensile stress. In the figures, a large change of the slope of the CTOA without consideration of residual stresses is caused by the change from the elastic response to the plastic response for some elements around the crack tip. When the applied stress is large, the CTOA with residual stresses is lower than that without residual stresses. The CTOA with residual stresses is larger than that without residual stresses when the applied tensile stress is less than $150 \mathrm{MPa}$ as shown in Fig. 13. This trend is quite similar to that of the values of $J_{\text {ld }}^{3}$ with and without residual stresses as shown in Figs. 10 and 12.

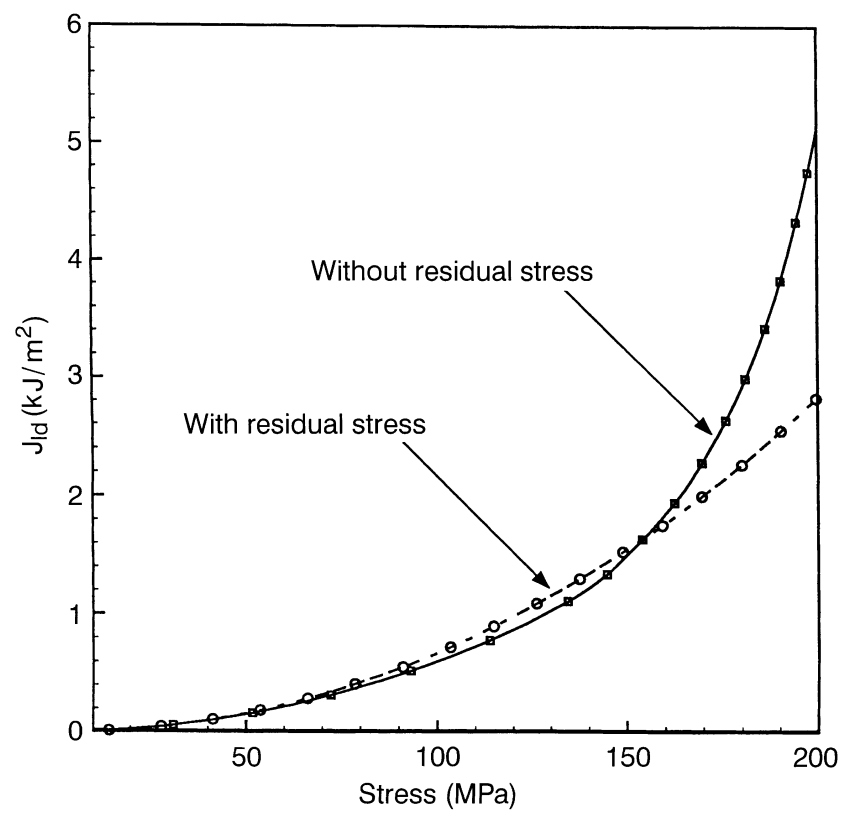

Fig. 12. The values of $J_{\mathrm{ld}}^{3}$ as function of the applied tensile stress with and without residual stresses

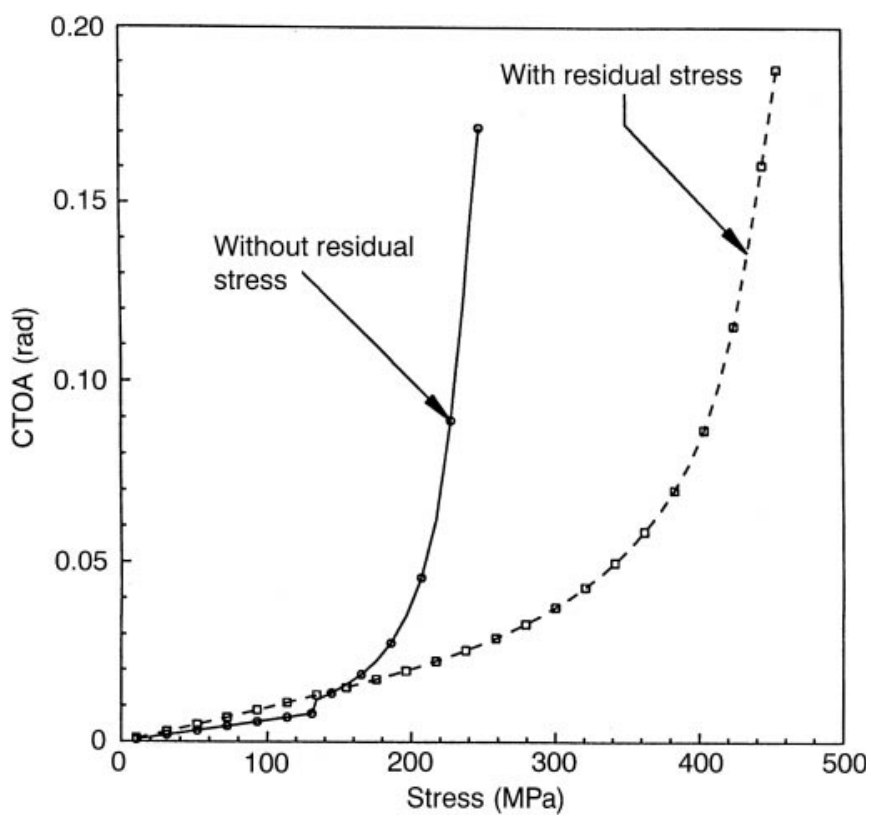

Fig. 13. The crack tip opening angles (CTOAs) as functions of the applied tensile stress with and without residual stresses

\section{4}

\section{Discussions and conclusions}

Most of the current computational works for fracture analyses of welded structures are based on the stress intensity factor $K$ of linear elastic fracture mechanics. For example, residual stresses from welding processes are usually calculated by thermoelastic-plastic analyses. The residual stresses are then used to calculate the linear elastic stress intensity factor $K$ without consideration of the effect of thermoplastic deformation history. The total value of $K$ at the crack tip is the sum of the value of $K$ due to the residual stresses and the value of $K$ due to the subsequent applied load. Sometimes, the value of $K$ due to the residual stresses is evaluated by taking the value of $K$ due to the remote tensile stress at, say, $20 \%$ of the yield stress.

Rigorously speaking, a logical fracture parameter for cracks in welds must be a local parameter such as the crack tip opening angle (CTOA) or the crack tip opening displacement (CTOD) obtained consistently with microscopically significant length scales to reflect the local and global residual stress effects on crack tip deformation and stresses. On the other hand, fracture parameters such as $J_{c}$ for cracks in welds and heat affected zones are determined by experiments. From the viewpoint of consistency with experimental data, we here present a computational scheme to calculate a potential fracture parameter $J_{\text {ld }}$ (the subscripts ld stands for load-displacement) for elasticplastic materials with residual stresses. The finite element computational scheme is similar to the virtual crack extension method for nonlinear elastic materials (Parks, 1977). We calculate the change of the work to the cracked structure based on the remote load-displacement curve due to small amount of crack growth. This concept is consistent with the determination of the experimental values of $J_{\mathrm{c}}$ for cracks in welds and heat affected zones from the load-displacement curves of the specimens cut out from welded structures. 
We have examined a case of two plates welded together by multi-pass welding processes. First, we calculate the residual stresses due to the welding processes and then we introduce a crack. Subsequently, we apply remote tensile stress on the welded plate. Our numerical results show that $J_{\text {ld }}$ 's for different contours close to the crack tip are in good agreement at the given applied stresses. Thus, $J_{\mathrm{ld}}$ may be considered as a potential fracture parameter for welded structures. Our finite element analyses show that the $J$ integral is quite path dependent when the residual stresses are considered. Thus, the $J$ integral cannot be used to characterize the crack-tip deformation processes. The numerical results also show that the values of $J_{\text {ld }}$ are lower for the welded plate at large applied tensile stresses when the residual stresses are considered.

Note that the trend of the local CTOA agrees well with the trend of $J_{\text {ld }}$ calculated from the global load-displacement curve. When we calculate $J_{\text {ld }}$, we perturb the finite element mesh near the crack tip and relate the change to the remote load-displacement curve. Therefore $J_{\mathrm{ld}}$ and CTOA has the same trend. $J_{\text {ld }}$ reduces to $J$ when no residual stress is considered. When the additional plastic deformation near the tip due to the applied load is large, the crack-tip stress and deformation fields due to the applied load become dominant. Then $J_{\text {ld }}$ regains its physical significance as a regular $J$ that controls the near-tip dominant singular stress and strain fields since the residual stress effects are washed out. However, more computational investigation is needed to understand the validity of $J_{l d}$ for modeling fracture processes due to cracks in welds and heat affected zones when the additional plastic deformation due to the applied load is moderate. Finally, $J_{\text {ld }}$ should be evaluated at a contour at a length scale related to the microstructure of materials. Further exploration of the applicability of $J_{\mathrm{ld}}$ is needed.

\section{References}

Atluri SN, Nishioka T, Nakagaki M (1984) Incremental PathIndependent Integrals in Inelastic and Dynamic Fracture Mechanics. Eng. Fract. Mech. 20:209-244

Atluri SN (1997) Structural Integrity and Durability. Tech-Science Press, Forsyth, Ga

Begley JA, Landes JD (1972a) The J-integral as a Fracture Criterion. Fracture Toughness, ASTM STP 514, American Society of Testing and Materials, Philadephia, 1-23
Begley JA, Landes JD (1972b) The Effect of Specimen Geometry on $J_{I C}$. Fracture Toughness, ASTM STP 514, American Society of Testing and Materials, Philadelphia, 24-39

Brust FW, McGown JJ, Atluri SN (1986) A Combined Numerical/ Experimental Study of Ductile Crack Growth after a Large Un-

loading, Using $T^{*}, J$ and CTOA Criteria. Eng. Fract. Mech. 23:537550

deLorenzi HG (1982) On the Energy Release Rate and the J-Integral of 3-D Crack Configurations. Int. J. Fract. 19:183-193

Hellen TK (1975) On the Method of Virtual Crack Extensions. Int. J. Numer. Methods Eng. 9:182-207

Hou Y-C, Kim M, Pan J, Brust FW (1996) Effects of Residual Stresses on Fracture of Welded Pipes. Residual Stresses in Design, Fabrication, Assessment, and Repair, PVP-Vol. 327, ASME, New York, 67-75

Moran B, Shih CF (1987) A General Treatment of Crack Tip

Contour Integrals. Int. J. Fract. 35:295-310

Nagtegaal JC, Parks DM, Rice JR (1974) On Numerically Accurate Finite Element Solutions in the Fully Plastic Range. Comp. Methods Appl. Mech. Eng. 4:153-177

Nakagaki M, Marschall, CW, Burst FW (1989) Elastic-Plastic Fracture Mechanics Evaluations of Stainless Steel Tungsten/InertGas Welds. Nonlinear Fracture Mechanics: Volume II-ElasticPlastic Fracture, ASTM STP 995, American Society for Testing and Materials, Philadelphia, 214-243

Nikishkov GP, Atluri SN (1987a) Calculation of Fracture Mechanics Parameters for Arbitrary Three-Dimensional Cracks by the Equivalent Domain Integral Method. Int. J. Num. Meth. Eng. 24:1801-1821

Nikishkov GP, Atluri SN (1987b) An Equivalent Domain Integral Method for Computing Crack-Tip Integral Parameters in NonElastic, Thermo Mechanical Fracture. Eng. Fract. Mech. 26:851867

Parks DM (1974) A Stiffness Derivative Finite Element Technique for Determination of Crack Tip Stress Intensity Factors. Int. J. Fract. 10:487-502

Parks DM (1977) The Virtual Crack Extension Method for Nonlinear Material Behavior. Comp. Methods Appl. Mech. Eng. 12:353-364

Rice JR (1968a) A Path Independent Integral and the Approximate Analysis of Strain Concentration by Notches and Cracks. J. App. Mech. 35:379-386

Rice JR (1968b) Mathematical Analysis in the Mechanics of Fracture. Fracture, Liebowitz, H. (ed), 2, New York, Academic Press, 191-131

Shih CF, Moran b, Nakamura T (1986) Energy Release Rate Along a Three-Dimensional Crack Front in a Thermally Stressed Body. Int. J. Fract. 30, 79-102

Wang L, Brust FW, Atluri SN (1997) "The Elastic-Plastic Finite Element Alternating Method (EPFEAM) and the Prediction of Fracture under WFD conditions in Aircraft Structures, Part II: Fracture and the $T^{*}$ Parameter" Comput. Mech. 19:370-379 